REVIEW

\title{
Psychosis in Parkinson's disease
}

\section{B R Thanvi, T C N Lo, D P Harsh}

Postgrad Med J 2005;81:644-646. doi: 10.1136/pgmj.2004.032029

Psychosis is common in Parkinson's disease (PD), particularly in its later stages. The symptoms range from comparatively minor illusions, vivid dreams, and occasional, non-disturbing visual hallucinations to frank psychosis. The pathogenesis of psychosis in PD is not fully known. Management of psychosis in PD requires a multidisciplinary approach. Some of the newer atypical antipsychotics are effective against psychosis with no significant worsening of PD. Psychosis in PD is associated with poor quality of life for patients and the carers.

See end of article for authors' affiliations

.....................

Correspondence to:

Dr B R Thanvi, Department

of Medicine for Older

People, Leicester General

Hospital, University

Hospitals of Leicester NHS

Trust, Gwendolen Road,

Leicester LE5 4PW, UK;

bthanvi@hotmail.com

Submitted

26 December 2004

Accepted 7 February 2005
$\mathrm{P}$ sychosis affects nearly one third of patients with Parkinson's disease (PD). ${ }^{1}$ Its manifestations range from comparatively minor symptoms of mild illusions, vivid dreams, and occasional, non-disturbing visual hallucinations in a clear sensorium to a frank psychosis with disturbing visual (and rarely, auditory and tactile) hallucinations, paranoid delusions, and confusional psychosis. Although it was a recognised complication even before L-dopa was introduced, psychosis has been increasingly reported in patients treated with dopaminergic drugs. Psychosis in PD significantly reduces the quality of life of the patients. It is the most important risk factor for transfer to a nursing home and is associated with a reduced survival. $^{2}$ It also results in increased stress for caregivers.

\section{EPIDEMIOLOGY AND RISK FACTORS}

Psychosis in PD generally occurs late in the disease, although there may be a bimodal onset, with early onset $(<5.5$ years $)$ associated with motor fluctuations and large doses of drugs, and more commonly a late onset ( $>5.5$ years) associated with cognitive impairment. ${ }^{3}$ Hospital based studies show prevalence rates of $8 \%-40 \%$ for hallucinations in patients receiving long term treatment for PD. ${ }^{4}$

Old age, cognitive impairment, history of depression, and sleep disorders are important risk factors for the development of psychosis in PD. ${ }^{5}$ Several drugs, including those used in treating PD (anticholinergic agents, selegiline, amantidine, dopamine agonists, catecholamine$o$-methyl transferase inhibitors, and L-dopa) can worsen or even precipitate psychosis in PD. The relation between the drug dose and psychosis in PD is complex. Underlying genetic susceptibility may be important. Interestingly, a recent study did not find antiparkinsonian drugs a risk factor for psychosis. ${ }^{6}$

\section{CLINICAL FEATURES}

Sleep disturbances (particularly rapid eye movement (REM) sleep disturbances), and vivid dreaming may precede hallucinations by weeks to months. ${ }^{7}$ Hallucinations are mostly visual and often non-threatening, at least initially. The visual hallucinations are mostly complex, usually containing animals, objects, or persons although simple hallucinations (characterised by the absence of form, for example, flashes of light or colour) also occur. They tend to occur in poor lighting conditions ("sundowning") or on waking from sleep. Patients may "see" crawling bugs on the patterned carpets or moving insects on the furniture.

Hallucinations usually occur after patients have taken PD drugs for several months or years. When hallucinations occur early in the course of presumed PD or when they occur shortly after the introduction of dopaminergic medicines, other diagnoses besides PD, for example, dementia with Lewy bodies or Alzheimer's disease should be considered. As hallucinations become more vivid, insight may be lost and the patient may start acting upon hallucinations. Auditory and tactile hallucinations may follow causing an organic confusional psychosis. A simple scale to assess the severity of hallucinations (table 1) can be used. ${ }^{8}$ Paranoid delusions, often directed towards the spouse or other family members, may be very distressing and often precipitate institutionalisation.

\section{PATHOPHYSIOLOGY}

Despite advances in neurochemistry, the pathophysiological basis of psychosis in PD remains poorly understood. Both exogenous (for example, drugs) and endogenous (related to the disease process itself) factors contribute to the development of psychosis in PD. The interplay between various neurotransmitters including dopamine, acetylcholine, and serotonin (5hydroxytryptamine, 5-HT) seems to be of importance. ${ }^{9}$ Dopamine has long been recognised as an important neurotransmitter in the disorders of perception. Dopamine agonists (for example, amphetamine) are known to produce psychosis and the commonly used antipsychotic agents are blockers of dopamine receptors. It is suggested that the dopaminergic overactivity in the pathways to limbic system and cerebral cortex is involved in the production of psychosis.

Degeneration of some of the pathways using 5HT as a neurotransmitter may play an important part. The improvement of psychosis with ondansetron (a 5-HT3 antagonist) and atypical

Abbreviations: PD, Parkinson's disease; REM, rapid eye movement; $A A$, atypical antipsychotic 


\begin{tabular}{ll}
\hline Table $1 \quad$ Hallucination scale in Parkinson's disease \\
\hline Symptom & Score \\
\hline None & 0 \\
Vivid dreams, illusions, sense of presence & 1 \\
Dreams encroaching on waking hours, occasional tolerable & 2 \\
hallucinations & \\
Regular evening and night time intrusive visual hallucinations & 3 \\
\hline
\end{tabular}

neuroleptics (with their blocking effects on serotonin receptors and dopamine receptors) lends some support to this concept.

Acetylcholine is another neurotransmitter with a potential role in psychosis of PD.

Pathological studies have shown significant degeneration of cholinergic neurons in PD. ${ }^{10}$ Goetz et al postulated that acetylcholine block could induce PD related hallucinations. ${ }^{11}$

As in many organic conditions associated with visual hallucinations, abnormalities of visual processing have also been implicated in the generation of hallucinations in PD. ${ }^{12}$ Parkinsonian patients with hallucinations had difficulty distinguishing between images they saw and images created in their mind. ${ }^{13}$ It has been shown that PD patients without hallucinations process visual information in the occipital/ temporal and parietal areas, whereas those with hallucinations do it in the frontal cortex. ${ }^{14}$

Sleep disturbances commonly precede psychosis in PD. Acetylcholine is an important neurotransmitter in the pathways controlling REM sleep. Some authors have suggested a link between hallucinations and perturbations of REM sleep. ${ }^{15}$

Two different mechanisms have been proposed for the two subgroups susceptible to hallucinosis in PD. ${ }^{3}$ In patients with disease duration of five years or less, postsynaptic dopamine receptor denervation supersensitivity in the mesolimbic/ mesocortical system may underlie hallucinosis. In patients with disease duration greater than five years, hallucinosis may be mediated either by changes outside the basal ganglia or by the serotonergic system.

It is probable that the psychosis in PD is multifactorial and is caused by abnormalities in several neurochemical transmitters and neural structures.

\section{MANAGEMENT}

Psychosis in PD poses great management challenge because of potentially catastrophic consequences for the patient and the carers, lack of universally effective and safe drugs, and the often progressive nature of this complication.

\section{General principles of management}

- It is vital to exclude delirium in all patients who present with psychosis. This is important as the treatment of the precipitating illness can potentially "cure" psychosis. A thorough search should be undertaken to exclude an intercurrent physical illness, drug effect, electrolyte imbalance, and systemic infection.

- A multidisciplinary team approach, involving patient, carer, physician, liaison psychiatrist, PD specialist nurse, and social worker is required for the effective management. Proper explanation of the nature and course of psychosis to the patient and carer helps them to cope with the situation.

- Simple measures should be tried first, for example, improving sleep hygiene, avoiding excessively patterned furniture, and reducing sensory overload and sensory deprivation.
- Psychiatric comorbidities of non-psychotic nature, for example, depression, anxiety, and apathy are common in PD patients with psychosis ${ }^{16}$ and may add to the functional impairment. Treatment of these conditions may reduce morbidity and distress to carers.

- Vivid dreams alone usually do not warrant medical treatment. Similarly, occasional visual hallucinations with insight retained may not require any action beyond reassurance.

- If simple measures fail, reduction in drug dose or elimination of one or more antiparkinsonian agents should be considered. The agent introduced most recently or one with the lowest antiparkinson efficacy (anticholinergics, amantadine, selegiline) should be stopped first. Next in order are dopamine agonists, catecholamine$o$-methyl transferase inhibitors, and L-dopa. It should be emphasised that dopamine agonists are much more likely to produce psychosis than L-dopa. One might have to accept worsening of parkinsonism.

\section{Drug treatment}

When the above measures fail, drug treatment becomes necessary. Drugs used to treat psychosis in PD include atypical antipsychotics (AA), 5-HT3 receptor antagonists, and acetylcholinesterase inhibitors (AChI).

(1) Atypical antipsychotics (AA): conventional antipsychotics (for example, haloperidol) cause intolerable worsening of motor function and should be avoided. Newer AA are associated with fewer extrapyramidal side effects because of their non-selective antagonism at both serotonergic and dopaminergic receptors. Examples of AA include clozapine, quetiapine, olanzapine, risperidone, and aripiprazole. The following factors should be carefully considered while using AA:

- With few exceptions, all AA have comparable efficacy against psychosis and the choice is mainly based on their ease of use and the side effect profile. Risperidone and olanzapine are associated with sedation and can cause considerable worsening of parkinsonism. Additionally, olanzapine can worsen cognition ${ }^{17}$ and hyperglycaemia in patients with diabetes. A recent CSM warning suggests an increased risk of strokes associated with the use of risperidone and olanzapine in old people. ${ }^{18}$ Clozapine is probably the most effective agent and does not cause worsening of parkinsonism. However, it is associated with the risk of potentially fatal, idiosyncratic agranulocytosis necessitating regular white blood cell counts. It has a restricted licence in the UK. Quetiapine is favoured by many psychiatrists because of its better side effect profile. Interestingly, a recent study showed quetiapine to be as effective as clozapine. ${ }^{19}$ Preliminary experience with the latest $\mathrm{AA}$, aripiprazole, has not been very encouraging, as it caused worsening of PD symptoms. ${ }^{20}$

- In older patients, AA can cause sedation and postural hypotension. Sedation can be helpful in patients whose behavioural problems occur at night when they are awake.

- AA should be started at very low doses with gradual increments if needed, as hypersensitivity to these agents can induce delirium or malignant neuroleptic syndrome. The doses required to treat psychosis are much lower than those used in schizophrenia.

- All AA cause prolongation of QT interval on ECG, particularly in older patients. Therefore, baseline ECG and periodic ECG monitoring are advisable.

- Optimal duration of treatment with AA in PD associated psychosis is not known. Because of the risk of serious side effects, long term use of AA should not be taken lightly. 
However, stopping AA in patients in remission is fraught with the possibility of recurrences and even "rebounds" of psychosis. Fernandez et al $^{21}$ conducted a prospective study on PD patients receiving successful treatment with quetiapine and clozapine as these drugs were withdrawn. After the antipsychotic agent was stopped, psychosis recurred in five of six patients. In three patients the "rebound psychosis" was worse than the original psychotic episode requiring higher antipsychotic drug doses.

- AA treatment improves prognosis in patients with psychosis. $^{22}$ In a study of 59 patients with psychosis treated with clazapine, the death rate was lower at $28 \%$ within two years compared with $100 \%$ in a study done before the availability of this treatment. Of all AA, clozapine is the most well studied agent and is considered as the "gold standard" treatment in psychosis of PD. In a randomised, placebo controlled trial, clozapine at a mean dose lower than $50 \mathrm{mg}$ /day improved drug induced psychosis in PD without significant worsening of motor function. ${ }^{23}$ The detailed account of individual AA in psychosis is beyond the scope of this article and interested readers are referred to a recent review. ${ }^{24}$

(2) Acetylcholinesterase inihibitors (AChI): these agents increase concentrations of acetylcholine in the brain and have been in use for treating Alzheimer dementia. Their use in psychosis of PD has been advocated on the basis of reports of beneficial effects in dementia with Lewy bodies. There is a significant loss of cholinergic neurons in PD and AChI may prove beneficial by increasing acetylcholine concentrations. In an open label study, rivastigmine led to improvement in cognitive and functional abilities, as well as the resolution of behavioural problems and visual hallucinations. ${ }^{25}$ Donepezil, another commonly used AChI, has also shown some promise $^{26}$ in open label studies.

(3) Ondensetron: it is a 5-HT3 antagonist widely used to treat vomiting attributable to anticancer agents. In an open label, short term (four to eight weeks) study, it was used to treat psychosis in PD at a dose of 12 to $24 \mathrm{mg}$ daily. ${ }^{27}$ There was pronounced to moderate improvement in measures of visual hallucinations, paranoid delusions, confusion, and the associated global functional impairment. It did not cause worsening in PD symptoms and was well tolerated. However, there have not been any large sized, controlled studies using ondensetron in PD associated psychosis. Ondensetron is an expensive drug.

Electroconvulsive therapy (ECT): ECT has been used in treating drug resistant depression in PD. There have also been reports of its success in improving motor symptoms of PD. However, it has not been systemically tested in treating psychosis of PD. There have been a few reports of its benefit in drug refractory psychosis of PD. ${ }^{28}$ Because of its cost and associated stigma, ECT is unlikely to be a primary treatment, except possibly for the drug resistant or drug intolerant cases or when psychosis is associated with severe depression.

\section{CONCLUSIONS}

Psychosis commonly complicates PD, especially in its later stages. It is a strong predictor of institutionalisation. Several risk factors have been identified including, old age, sleep disturbances, long duration of PD, history of depression, cognitive impairment, and visual disorders. Pathophysiology of psychosis remains unknown, although dopamine, serotonin, and $t$ acetylcholine are the major neurotransmitters of interest. Effective treatment of psychosis requires multidisciplinary team approach and attention to the intercurrent illnesses, optimising drug treatment of $\mathrm{PD}$, and judicious use of AA. Further studies are needed to evaluate the use of
5-HT3 antagonists, acetylcholinesterase inhibitors, and ECT in psychosis of PD.

\section{Authors' affiliations}

B R Thanvi, T C N Lo, Department of Medicine for Older People, Leicester General Hospital, University Hospitals of Leicester NHS Trust, Leicester, UK

D P Harsh, Hellesdon Hospital, Norwich, UK

Funding: none.

Conflicts of interest: none.

\section{REFERENCES}

1 Naimark D, Jackson E, Rockwell E, et al. Psychotic symptoms in Parkinson's disease patients with dementia. J Am Geriatr Soc 1996;44:296-9.

2 Goetz CG, Stebbins GT. Risk factors for nursing home placement in advanced Parkinson's disease. Neurology 1993;43:2227-9.

3 Graham JM, Grunwald RA, Sagar HJ. Hallucinations in idiopathic Parkinson's disease. J Neurol Neurosurg Psychiatry 1997;63:434-40.

4 Barnes J, David AS. Visual hallucinations in Parkinson's disease: a review and phenomenological survey. J Neurol Neurosurg Psychiatry 2001;70:727-33.

5 Sanchez-Ramos JR, Ortoll R, Paulson GW. Visual hallucinations associated with Parkinson's disease. Arch Neurol 1996;53:1265-8.

6 Merims D, Shabtai H, Korczyn AD, et al. Antiparkinsonian medication is not a risk factor for the development of hallucinations in Parkinson's disease. J Neural Transm 2004;111:1447-53.

7 Thanvi BR, Munshi SK, Vijaykumar N, et al. Neuropsychiatric non-motor aspects of Parkinson's disease. Postgrad Med J 2003;79:561-5.

8 Hindle JV. In Parkinson's disease in the older patient. In: Playfer JR, Hindle JV, eds. Neuropsychiatry. London: Arnold, 2001:124.

9 Birkmayer W, Riedere P. Responsibility of extrastriatal areas for the appearance of psychotic symptoms. J Neural Transm 1975;37:175-82.

10 Whitehouse PJ. Clinical and neurochemical consequences of neuronal loss in the nucleus basalis of Meyernert in Parkinson's disease and Alzheimer's disease. Adv Neurol 1986;45:393-6.

11 Goetz CG, Tanner CM, Klawans MD. Pharmacology of hallucinations induced by long-term drug therapy. Am J Psychiatry 1982;139:494-7.

12 Bodis-Wollner I. Neuropsychological and perceptual defects in Parkinson's disease. Parkinsonism Relat Disord 2003;9:S83-9.

13 Barnes J, Boubert L, Harris J, et al. Reality monitoring and visual hallucinations in Parkinson's disease. Neuropsychologia 2003;41:565-74.

14 Goetz CG. Hallucinations and parkinsonism. Seventeenth annual symposia on etiology, pathogenesis, and treatment of Parkinson's disease and other movement disorders, Parkinson Study Group, 19 Oct, 2003.

15 Kulisevsky J, Roldan E. Hallucinations and sleep disturbances in Parkinson's disease. Neurology 2004;63:S28-30.

16 Marsh L, Williams JR, Rocco M, et al. Psychiatric comorbidities in patients with Parkinson's disease and psychosis. Neurology 2004;63:293-7.

17 Graham JM, Sussman JD, Ford KS, et al. Olanzapine in the treatment of hallucinosis in idiopathic Parkinson's disease: a cautionary note. J Neurol Neurosurg Psychiatry 1998;65:774-7.

18 Committee on Safety of Medicines. Latest news. 9 Mar 2004. Atypical antipsychotic drugs and stroke. http://www.mca.gov.uk/aboutagency/ regframework/csm/csmhome.htm.

19 Morgante L, Epifanio A, Spina E, et al. Quetiapine and clozapine in parkinsonian patients with dopaminergic psychosis. Clin Neuropharmacol 2004;27:153-6.

20 Fernandez HH, Trieschmann ME, Friedman JH. Aripiprazole for drug-induced psychosis in Parkinson disease: preliminary experience. Clin Neuropharmacol 2004;27:4-5.

21 Fernandez HH, Trieschmann ME, Okun MS. Rebound psychosis: effect of discontinuation of antipsychotics in Parkinson's disease. Mov Disord 2005;20:104-5.

22 Factor SA, Feustel PJ, Friedman JH, et al. Longitudinal outcome of Parkinson's disease patients with psychosis. Neurology 2003;60:1756-61.

23 Pollak $\mathrm{P}$, Tison $\mathrm{F}$, Rascol O, et al. Clozapine in drug induced psychosis in Parkinson's disease: a randomised, placebo controlled study with open follow up. J Neurol Neurosurg Psychiatry 2004;75:689-95.

24 Wint DP, Okun MS, Fernandez HH. Psychosis in Parkinson's disease. J Geriatr Psychiatry Neurol 2004;17:127-36.

25 Bullock R, Cameron A. Rivastigmine for the treatment of dementia and visual hallucinations associated with Parkinson's disease: a case series. Curr Med Res Opin 2002;18:258-64.

26 Bergman J, Lerner V. Successful use of donepezil for the treatment of psychotic symptoms in patients with Parkinson's disease. Clin Neuropharmacol
2002;25:107-110.

27 Zoldan J, Friedberg G, Livneh M, et al. Psychosis in advanced Parkinson's disease: treatment with ondansetron, a 5-HT3 receptor antagonist. Neurology 1995;45:1305-8

28 Hoflich G, Burghof KW, Kasper S, et al. Electroconvulsive therapy in comorbidity of treatment refractory paranoid hallucinatory psychoses with Parkinson's disease. Nervenarzt 1994;65:202-5. 